\title{
2-氮杂双环[3.1.0]己烷-3-羧酸的不对称合成
}

\author{
付行花樊俭俭邹爱宗 俞娟何广科朱军* \\ (南京工业大学理学院 南京 211816)
}

\begin{abstract}
摘要 设计了以 $D$-谷氨酸或 $L$-谷氨酸为原料, 经氨基保护、4-二甲氨基吡定(DMAP)催化环合、还原脱水成烯、不对

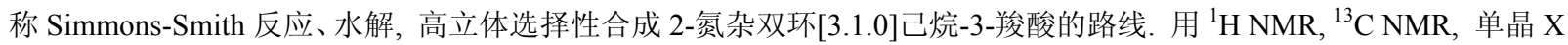
衍射等对中间体及目标产物进行了结构表征. 优化了 DMAP 催化环合、Simmons-Smith 和水解反应的工艺条件, 结果 表明, DMAP 催化环合反应在原料比为 $n$ (DMAP) $: n$ (二碳酸二叔丁酯) $: n$ (吡啶) $=0.40: 4.0: 1.0$ 条件下, $(R)-5$-氧代吡 咯-1,2-二甲酸二叔丁酯的收率可达 $82 \%$; Simmons-Smith 反应随着反应时间延长, 顺式结构与反式结构含量比不断变 化，直到反应时间为 $19.5 \mathrm{~h}$ 时，顺式与反式达到最佳比为 6: 1, 收率为 $75 \%$. 总收率达 $30 \%, d e$ 值 $72 \%$. 关键词 2-氮杂双环 [3.1.0]己烷-3-羧酸; 谷氨酸; DMAP 催化环合; 水解反应; 不对称合成
\end{abstract}

\section{Asymmetric Synthesis of 2-Azabicyclo[3.1.0]hexane-3- carboxylic Acid}

\author{
$\mathrm{Fu}$, Xinghua $\quad$ Fan, Jianjian $\quad$ Zou, Aizong $\quad$ Yu, Juan $\quad$ He, Guangke $\quad$ Zhu, Hongjun* \\ (College of Sciences, Nanjing Tech University, Nanjing 211816)
}

\begin{abstract}
Azabicyclo[3.1.0]hexane-3-carboxylic acids were obtained via amino protection, 4-dimethylaminopyridine (DMAP) catalytic cyclization, reduction-dehydration into alkene, asymmetric Simmons-Smith reaction and hydrolysis reaction from glutamic acid. The intermediates and target products were characterized by ${ }^{1} \mathrm{H}$ NMR and ${ }^{13} \mathrm{C}$ NMR. $(1 R, 3 S, 5 R)-$ 2-(tert-Butoxycarbonyl)-2-azabicyclo[3.1.0] hexane-3-carboxylic acid and (1S,3S,5S)-2-(tert-butoxycarbonyl)-2-azabicyclo[3.1.0]hexane-3-carboxylic acid were analyzed by X-ray diffraction as well. The process conditions about DMAP catalytic cyclization reaction, Simmons-Smith reaction and hydrolyzation were discussed. The results show that the yield of (R)-1,2-di-tert-butyl 5-oxopyrrolidine- 1,2-dicarboxyate was $82 \%$ at the ratio of $n$ (DMAP) $: n$ (di-tert-butyl dicarbonate) : $n$ (pyridine $)=0.40: 4.0: 1.0$. The ratio of cis/trans-form varied with the reaction time in Simmons-Smith reaction, which gave a best ratio of $6: 1$ at $19.5 \mathrm{~h}$. The total yield is $30 \%$, and the de value is $72 \%$.
\end{abstract}

Keywords 2-azabicyclo[3.1.0]hexane-3-carboxylic acid; glutamic acid; DMAP catalytic cyclization; hydrolysis reaction; asymmetric synthesis

肝炎即肝脏的肿胀或炎症, 导致肝炎的主要原因是 感染丙型肝炎病毒, 全球近 1.7 亿人感染了慢性丙型肝 炎病毒，但在丙型肝炎对肝脏造成严重损害和后果前， 丙型肝炎病毒感染者可能并没有任何症状 ${ }^{[1]}$. 西方发达 国家, 丙肝已成为肝移植的重要原因, 在确诊的丙型肝 炎肝硬化中肝癌发病率以每年 $1 \% \sim 4 \%$ 的幅度递增, 因 此开发新的治疗丙型肝炎药物成为医药科研人员研究 的热点 ${ }^{[2]}$. 百时美施贵宝公布的新型治疗丙肝药物分子 结构 BMS-1 ${ }^{[3]}$, 其两端是由不同构型的 2-氮杂双环 [3.1.0]己烷类化合物连接咪唑环通过中间桥基形成一个
对称的分子结构; 类似分子结构也于多篇专利中被报 导, 如专利 WO 099527 $7^{[4]}$ 公开了新型丙型肝炎药物的 结构式 $\mathbf{M}_{1}$ 和 $\mathbf{M}_{\mathbf{2}}$; 专利 WO 117635 $5^{[5]}$ 公开了丙型肝炎药 物中间体通式 $\mathrm{M}_{3}$; 专利 WO $052850^{[6]}$ 公开了一类用于 抑制二肽基肽酶类药物结构 $\mathbf{M}_{\mathbf{4}}$, 不难看出 2-氮杂双环 [3.1.0]己烷-3-羧酸化合物是这些新型药物关键中间体.

基于 2-氮杂双环[3.1.0]己烷-3-羧酸化合物的重要 性，一些专利对其合成作了一定的报道，如 2004 年 $\mathrm{Vu}$ 等 ${ }^{[6]}$ 以 $L$-焦谷氨酸为原料合成了中间体 $(1 S, 3 S, 5 S)-2$-叔 丁氧羰基-2-氮杂双环[3.1.0]己烷-3-甲酸(R1')；2008 年

\footnotetext{
*E-mail: zhuhj@njtech.edu.cn

Received March 3, 2014; revised March 31, 2014; published online April 18, 2014.

Project supported by the National Key Technology R\&D Program of the Ministry of Science and Technology (No. 2011BAE06A07-04).

国家 “十二五科技支撑计划” (No. 2011BAE06A07-04)资助项目.
} 


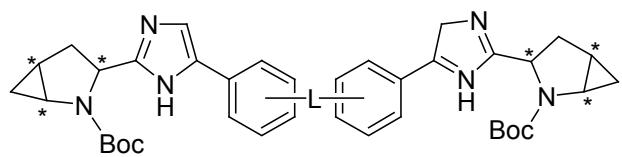

BMS-1<smiles>[R]N1C(c2ncc(-c3ccc(-c4cnc5[nH]c(C6CC7CC7N6[R])nc5c4)cc3)[nH]2)CC2CC21</smiles><smiles></smiles><smiles></smiles>

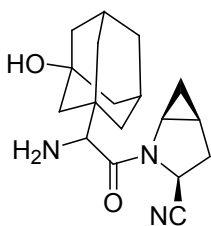

$\mathbf{M}_{4}$

图 1 代表性的含氮杂双环己烷结构单元的化合物

Figure 1 Representative compounds containing azabicyclo[3.1.0]hexane structure unit

Aissaoui 等 ${ }^{[7]}$ 以 $L$-焦谷氨酸乙酯为原料合成了中间体 $(1 S, 3 S, 5 S)$-2-氮杂双环[3.1.0]己烷-2,3-二甲酸-2-叔丁酯3 -乙酯; 2012 年 Neitz 等 $^{[8]}$ 以 $D$-焦谷氨酸为原料合成了 中间体(3R)-2-氮杂双环[3.1.0]己烷-2,3-二甲酸-2-叔丁 酯-3-乙酯; 2013 年 Borriellom 等 ${ }^{[9]}$ 以 $D$-焦谷氨醇为原料 合成了中间体(3R)-2-氮杂双环[3.1.0]己烷-2,3-二甲酸二 叔丁酯. 这几篇专利中对化合物 2-氮杂双环[3.1.0]己烷3-羧酸的合成均存在着操作复杂, 反应步骤多, 收率不 高等缺点. 因此本文在优化上述文献 $[6 \sim 9]$ 的基础上, 设计了一条以谷氨酸为原料经氨基保护, 环合, 还原, 西蒙斯一史密斯反应, 水解, 高收率、高立体选择性合成 2-氮杂双环[3.1.0]己烷-3-羧酸的路线 (以 $D$-谷氨酸为原 料的合成路线如图 2 所示, 以 $L$-谷氨酸为原料的合成路 线与其类似).

\section{1 结果与讨论}

\subsection{DMAP 催化环合的研究}

目前文献报道的合成 5-氧代吡咯-1,2-二甲酸酯的 方法主要是先合成焦谷氨酸酯, 再经二碳酸二叔丁酯
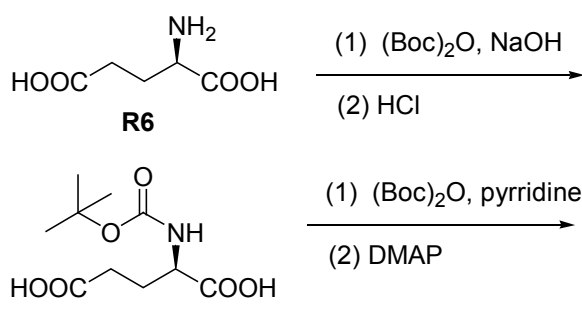

R5
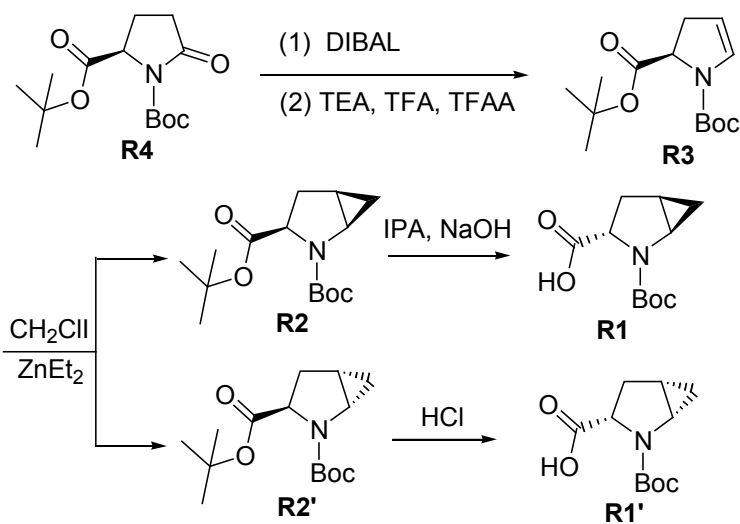

图 2 2-氮杂双环[3.1.0]己烷-3-羧酸的合成路线

Figure 2 The synthetic route of 2-azabicyclo[3.1.0]hexane-3carboxylic acid

(Boc)保护氨基得到. 而合成焦谷氨酸酯主要有如图 3 所 示的两条途经 ${ }^{[10]}$, 其不同点在于酯化和环合的次序. 我 们研究发现这两种方法条件不易控制，高温环合这步容 易消旋化和碳化. 而 4-二甲氨基吡啶(DMAP)是一种新 型高效催化剂，广泛用于催化酰化、酰胺化、酯化、硅 烷化等反应 ${ }^{[11]}$. 与传统酰化催化剂相比, DMAP 催化酰 化反应有反应温度低、反应速率快、收率高等优点，特 别是对空间位阻大的酰化反应有独特的效果 ${ }^{[12]}$. 使用 DMAP 时, 一般加入吡啶或者三乙胺作为助催化剂, 以 减少 DMAP 的用量 ${ }^{[13]}$. 为此, 我们先用 Boc 保护谷氨酸 中的氨基，再以 DMAP 为环合催化剂，催化环合得到 5-氧代吡咯-1,2-二甲酸二叔丁酯(R4). 考察了 $n($ Boc- $D-$ 谷氨酸)：n(DMAP)：n(Boc)对 5-氧代吡咯-1,2-二甲酸 二叔丁酯收率的影响, 结果见表 1 . 由表 1 Entries $1 \sim 6$ 可知: $n$ (DMAP) : $n$ (Boc- $D$-谷氨酸)的物质的量比由 $0.20: 1.0$ 增加到 $0.50: 1.0$, 产品的收率先增加后减少, 当物质的量比在 $0.40: 1.0$ 时, 产品收率达到最佳值 $80 \%$; 表 1 Entries 7 12 可知, 随着 Boc 用量的增加, 产 品收率逐渐增高, 当 $n(\mathrm{BOC}): n(\mathrm{Boc}-D$-谷氨酸 $)=4.0$ : 1.0 时，收率达到最佳值 $82 \%$.

\section{2 不对称 Simmons-Smith 反应的研究}

Simmons-Smith 反应是立体专一的顺式加成，通 常会受到位阻效应的影响, 反应在双键位阻较小的一侧

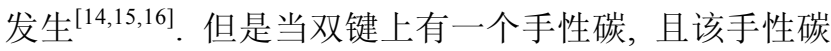




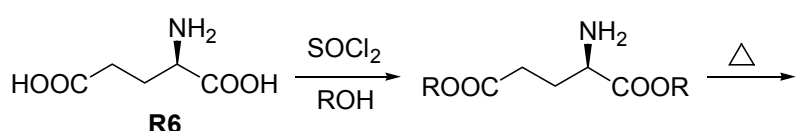<smiles>[R]OC(=O)[C@H]1CCC(=O)N1</smiles><smiles>[R6]OC(=O)C(N)CCC(=O)O</smiles><smiles>[R]OC(=O)[C@H]1CCC(=O)N1</smiles>

$\mathrm{R}=\mathrm{Me}, \mathrm{Et}$

图 3 文献报道的合成路线

Figure 3 The reported synthetic route

表 1 原料 Boc- $D$-谷氨酸(R5)与试剂物质的量比对 $(R)-5$-氧代 吡咯- 1,2-二甲酸二叔丁酯(R4)收率的影响

Table 1 Effect of the molar ratio of Boc- $D$-glutamic acid (R5) with reagents on the yield of $(R)$-1,2-di-tert-butyl 5-oxopyrrolidine-1,2-dicarboxyate (R4)

\begin{tabular}{ccc}
\hline Entry & $n($ R5) $: n($ DMAP) $: n$ (Boc) & Yield\% \\
\hline 1 & $1.0: 0.20: 4.0$. & 36 \\
2 & $1.0: 0.30: 4.0$ & 38 \\
3 & $1.0: 0.35: 4.0$ & 44 \\
4 & $1.0: 0.40: 4.0$ & 80 \\
5 & $1.0: 0.45: 4.0$ & 45 \\
6 & $1.0: 0.50: 4.0$ & 43 \\
7 & $1.0: 0.40: 2.0$ & 41 \\
8 & $1.0: 0.40: 3.0$ & 53 \\
9 & $1.0: 0.40: 3.5$ & 56 \\
10 & $1.0: 0.40: 4.0$ & 82 \\
11 & $1.0: 0.40: 4.5$ & 65 \\
12 & $1.0: 0.40: 5.0$ & 64 \\
\hline
\end{tabular}

上又连有羟基取代基时，由于羟基可与锌配位，反应往 往在羟基同侧发生 ${ }^{[17]}$.

参考 Simmons-Smith 反应 ${ }^{[18,19]}$, 本文由原料 $\mathbf{R 3}$ 和 $\mathrm{CH}_{2} \mathrm{ClI}$ 及 $\mathrm{Et}_{2} \mathrm{Zn}$ 高立体选择性合成得到化合物 $\mathbf{R} 2$ 和 R2'. 其推测反应机理如图 4 所示. 二乙基锌先与氯碘甲 烷生成化合物 $\mathrm{EtZnCH}_{2} \mathrm{Cl}, \mathrm{Zn}$ 可与 $\mathbf{R 3}$ 手性碳所连的羰 基氧配位，将有关基团针定在羰基的同侧，因此亚甲基 加成于叔丁氧羰基的同侧, 生成顺式产物. 由于 $\mathrm{Zn}$ 与 羰基的作用没有与羟基的作用强, 及手性碳的另一侧空 间位阻较小，因此也会有少量反式产物 R2'产生. 同时 发现反应时间对该反应的立体选择性有一定影响，用 HPLC 监控该反应的结果如图 5 所示. 当反应时间到 $19.5 \mathrm{~h}$ 时两者之间的比例达到最佳为 $6: 1$, 顺式占主体 部分.

同时，我们也考察了温度对于反应结果的影响. Simmons-Smith 反应中用到的试剂二乙基锌比较活泼, 一般需要加入配体或者辅助试剂 ${ }^{[20]}$, 由此我们考察了

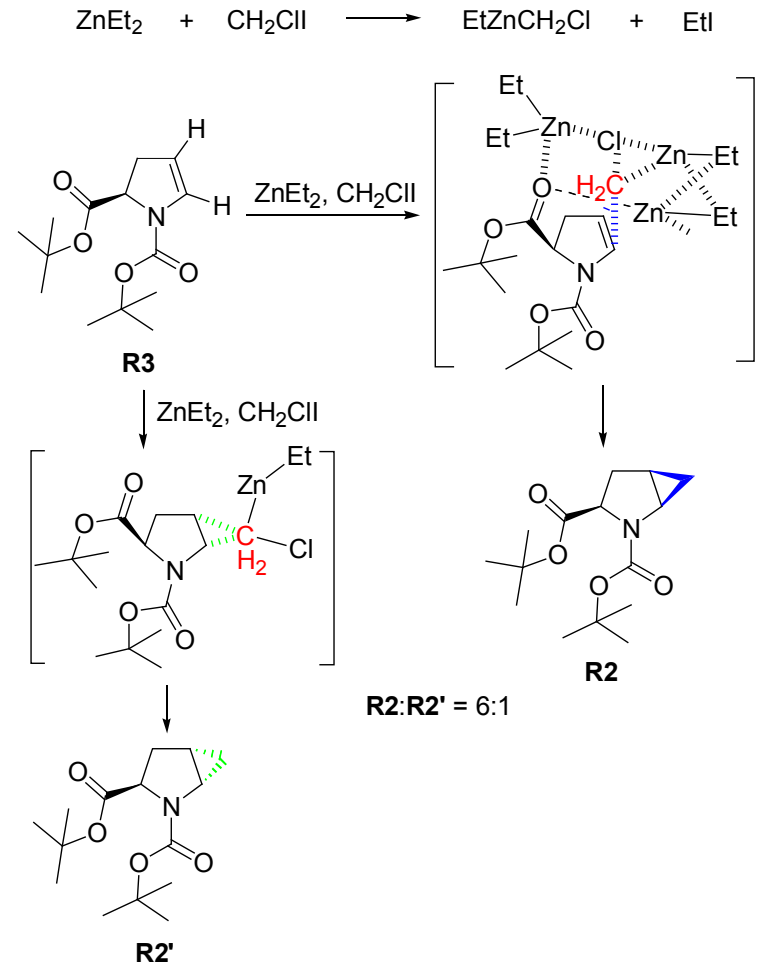

图 4 推测 Simmons-Smith 反应历程

Figure 4 Possible paths for the Simmons-Smith reaction

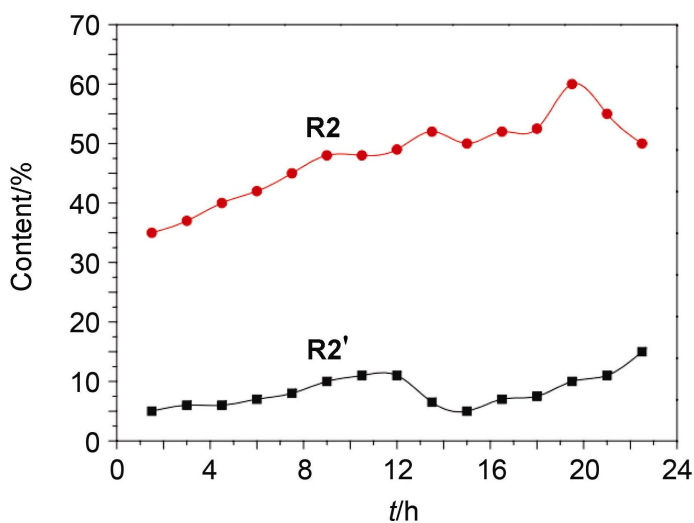

图 5 中间体 $\mathbf{R 2}$ 和 $\mathbf{R 2}$ '含量随反应时间变化的曲线

Figure 5 The content of intermediates $\mathbf{R 2}$ and $\mathbf{R 2}$ ' varied with the reaction time

$-30,-20$ 和 $-10{ }^{\circ} \mathrm{C}$ 时反应情况. 当温度为 $-30{ }^{\circ} \mathrm{C}$ 时, 原料 $\mathbf{R 3}$ 在 $30 \mathrm{~h}$ 时才反应完全, 顺式产物与反式产物比 为 $6: 1$; 温度为 $-20{ }^{\circ} \mathrm{C}$ 时, 在 $19.5 \mathrm{~h}$ 时原料 $\mathbf{R 3}$ 反应完 全，顺式产物与反式产物比为 $6: 1$; 温度为 $-10{ }^{\circ} \mathrm{C}$ 时, 在 $15 \mathrm{~h}$ 时原料 $\mathbf{R 3}$ 反应完全, 但是副产物较多, 所需的 产物含量很少. 结果表明当反应温度在零下 $20{ }^{\circ} \mathrm{C}$ 时, 相应的产率和立体选择性达到最佳.

\subsection{2 -氮杂双环[3.1.0]己烷-2,3-二甲酸二叔丁酯 $(R 2$ 和 R2')的水解}

氢氧化钠是常用的酯水解试剂，早在 1979 年, Hall 
等 ${ }^{[21]}$ 已经对以氢氧化钠为碱性试剂对磷酰胺类化合物 的立体选择性水解有了一定研究, 之后, 于 1986 年唐除 疾等 ${ }^{[22]}$ 研究旋光性硫代磷酰氯的碱性水解过程中发现, 其磷原子构型发生了翻转; 此后, 于 1992 年唐除痴 等 ${ }^{[23]}$ 研究了不同氨基磷酰胺的水解, 结果证明该类化 合物碱性水解的立体化学为构型翻转; 1996 年 Parr 等 ${ }^{[24]}$ 在吡咯环上甲酸甲酯的碱性水解过程中发现化合物发 生了差向异构化.

由于化合物 $\mathbf{R 2}$ 和 $\mathbf{R 2} \mathbf{2}^{\prime}$ 结构中含有的 Boc 在酸性条 件下易发生水解, 为此对其在氢氧化钠溶液中的水解情 况进行了研究, 水解后得到产物 R1 和 $\mathbf{R} 1{ }^{\prime}$, 它们的比例 为 $4:$ 1. 结合得到 $\mathbf{R} 1$ 和 $\mathbf{R} 1{ }^{\prime}$ 的单晶分析结果(见第 1.4 节)和第 1.2 节的讨论, 可以看出有关化合物在水解过程 中羧基的 $\alpha$-碳可能发生了构型翻转, 即构型为 $(1 R, 3 R, 5 R)$-2-氮杂双环[3.1.0]己烷-2,3-二甲酸二叔丁酯 (R2) 水解得到产物( $1 R, 3 S, 5 R)$-2-叔丁氧羰基-2-氮杂双环 [3.1.0]己烷-3-甲酸 $(\mathbf{R 1})$; 构型为 $(1 S, 3 R, 5 S)$-2-氮杂双环 [3.1.0]己烷-2,3-二甲酸二叔丁酯 (R2') 水解得到产物 $(1 S, 3 S, 5 S)$-2-叔丁氧羰基-2-氮杂双环[3.1.0]己烷-3-甲酸 $\left(\mathbf{R 1}^{\prime}\right)$. 所测化合物 R1, R1'，(1S,3R,5S)-2-叔丁氧羰基-2氮杂双环[3.1.0]己烷-3-甲酸 $(\mathbf{S 1})$ 和 $(1 R, 3 R, 5 R)$-2-叔丁氧 羰基-2-氮杂双环 [3.1.0] 已烷-3-甲酸(S1') 的比旋光度分 别为 $[\alpha]_{\mathrm{D}}^{12}-80.2,-43.7,+85.5,+42.1$ (c 0.2, $\mathrm{CH}_{3}-$ $\left.\mathrm{CH}_{2} \mathrm{OH}\right)$. 其中 $\mathbf{R} 1$ 与 $\mathbf{S 1}, \mathbf{R} \mathbf{1}^{\prime}$ 与 $\mathbf{S} 1^{\prime}$ 分别是对映体, 有关 结果也证明了所推测水解过程中羧基所在 $\alpha$-碳发生了 构型翻转. 有关构型翻转机理还有待进一步研究.

\section{$1.4 \mathrm{R} 1$ 与 R1'的晶体结构}

为了进一步确定手性 2-氮杂双环 [3.1.0]己烷-3-羧酸 $\mathrm{R} 1$ 和 R1'的结构, 我们用 $\mathrm{X}$ 射线单晶衍射方法对 $\mathrm{R} 1$ 和 $\mathbf{R} 1^{\prime}$ ' $^{\prime}$ 结构进行了分析. R1 的晶体结构如图 6 所示, 分

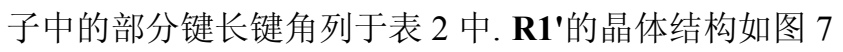
所示, 分子中的部分键长键角列于表 3 中.

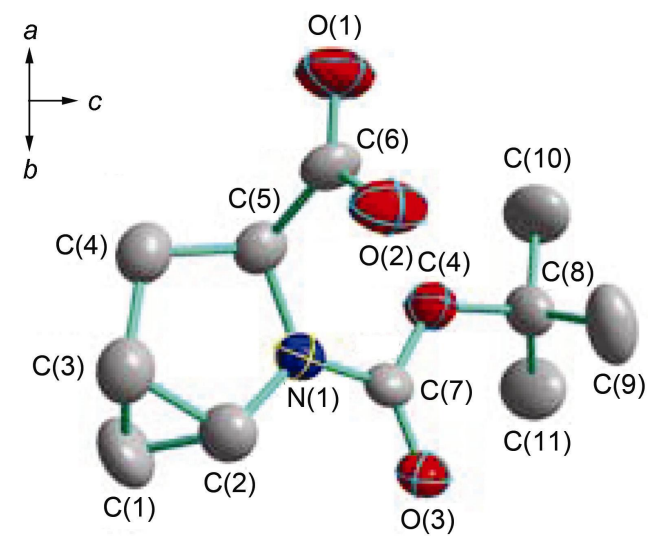

图 $6 \mathrm{R1}$ 的晶体结构

Figure 6 Crystal structure of R1

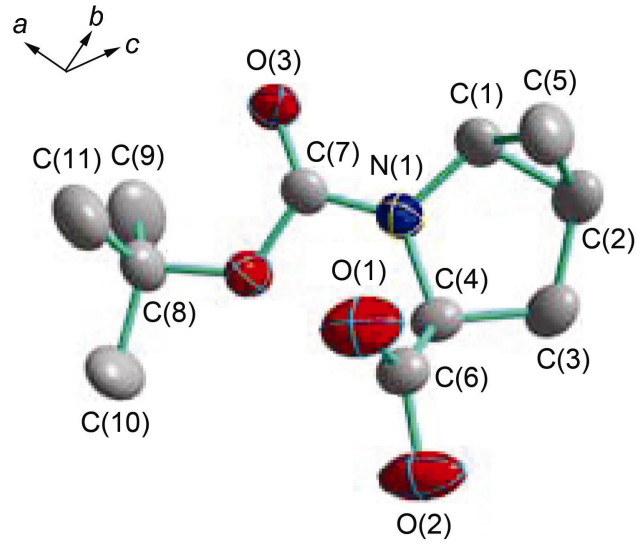

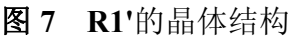

Figure 7 Crystal structure of R1'

表 2 R1 的部分键长和键角

Table 2 Selected bond lengths and angles for R1

\begin{tabular}{|c|c|c|}
\hline \multicolumn{3}{|c|}{ Bond length/Å } \\
\hline $\mathrm{N}-\mathrm{C}(7)$ & $1.336(4) \mathrm{O}(3)-\mathrm{C}(7)$ & $1.220(3)$ \\
\hline $\mathrm{N}-\mathrm{C}(1)$ & $1.437(4) \mathrm{C}(3)-\mathrm{C}(4)$ & $1.533(4)$ \\
\hline $\mathrm{N}-\mathrm{C}(4)$ & $1.459(4) \mathrm{O}(4)-\mathrm{C}(7)$ & $1.337(3)$ \\
\hline $\mathrm{O}(1)-\mathrm{C}(6)$ & $1.183(3) \mathrm{O}(4)-\mathrm{C}(8)$ & $1.472(4)$ \\
\hline$C(1)-C(2)$ & $1.479(5) \quad \mathrm{C}(8)-\mathrm{C}(11)$ & $1.491(5)$ \\
\hline $\mathrm{O}(2)-\mathrm{C}(6)$ & $1.317(4) \quad C(2)-C(3)$ & $1.519(5)$ \\
\hline \multicolumn{3}{|c|}{ Bond angle/( $\left(^{\circ}\right)$} \\
\hline $\mathrm{C}(7)-\mathrm{N}-\mathrm{C}(2)$ & 122.2(3) $\mathrm{N}-\mathrm{C}(5)-\mathrm{C}(4)$ & 104.2(3) \\
\hline$C(7)-N-C(5)$ & $124.3(3) \mathrm{C}(6)-\mathrm{C}(5)-\mathrm{C}(4)$ & $110.4(3)$ \\
\hline $\mathrm{C}(2)-\mathrm{N}-\mathrm{C}(5)$ & $113.4(3) \quad \mathrm{N}-\mathrm{C}(5)-\mathrm{H}(5 \mathrm{~A})$ & 109.4 \\
\hline$C(2)-C(1)-C(3)$ & $59.2(3) \mathrm{O}(3)-\mathrm{C}(7)-\mathrm{N}$ & $124.4(4)$ \\
\hline $\mathrm{N}-\mathrm{C}(2)-\mathrm{C}(3)$ & $106.5(4) \mathrm{O}(4)-\mathrm{C}(8)-\mathrm{C}(10)$ & $102.0(3)$ \\
\hline $\mathrm{N}-\mathrm{C}(2)-\mathrm{C}(1)$ & 115.3(4) $\mathrm{C}(9)-\mathrm{C}(8)-\mathrm{C}(10)$ & $111.9(4)$ \\
\hline $\mathrm{N}-\mathrm{C}(5)-\mathrm{C}(6)$ & $113.9(3) \quad \mathrm{O}(4)-\mathrm{C}(8)-\mathrm{C}(11)$ & $110.9(4)$ \\
\hline
\end{tabular}

表 3 R1'的部分键长和键角

Table 3 Selected bond lengths and angles for R1'

\begin{tabular}{lll}
\hline \multicolumn{4}{c}{ Bond length/ $\AA$} \\
\hline $\mathrm{N}-\mathrm{C}(7)$ & $1.336(4) \mathrm{O}(3)-\mathrm{C}(7)$ & $1.220(3)$ \\
$\mathrm{N}-\mathrm{C}(1)$ & $1.437(4) \mathrm{C}(3)-\mathrm{C}(4)$ & $1.533(4)$ \\
$\mathrm{N}-\mathrm{C}(4)$ & $1.459(4) \mathrm{O}(4)-\mathrm{C}(7)$ & $1.337(3)$ \\
$\mathrm{O}(1)-\mathrm{C}(6)$ & $1.183(3) \mathrm{O}(4)-\mathrm{C}(8)$ & $1.472(4)$ \\
$\mathrm{C}(1)-\mathrm{C}(2)$ & $1.479(5) \mathrm{C}(8)-\mathrm{C}(11)$ & $1.491(5)$ \\
$\mathrm{C}(1)-\mathrm{C}(5)$ & $1.488(5) \mathrm{C}(2)-\mathrm{C}(3)$ & $1.519(5)$ \\
\hline \multicolumn{5}{c}{ Bond angle/( $\left.{ }^{\circ}\right)$} \\
$\mathrm{C}(7)-\mathrm{N}-\mathrm{C}(1)$ & $122.8(3) \mathrm{N}-\mathrm{C}(4)-\mathrm{C}(3)$ \\
$\mathrm{C}(7)-\mathrm{N}-\mathrm{C}(4)$ & $124.7(2) \mathrm{O}(3)-\mathrm{C}(7)-\mathrm{N}$ \\
$\mathrm{C}(1)-\mathrm{N}-\mathrm{C}(4)$ & $112.3(2) \mathrm{O}(3)-\mathrm{C}(7)-\mathrm{O}(4)$ & $104.2(2)$ \\
$\mathrm{N}-\mathrm{C}(1)-\mathrm{C}(2)$ & $107.4(3) \mathrm{N}-\mathrm{C}(7)-\mathrm{O}(4)$ & $125.2(3)$ \\
$\mathrm{N}-\mathrm{C}(1)-\mathrm{C}(5)$ & $116.4(3) \mathrm{O}(4)-\mathrm{C}(8)-\mathrm{C}(11)$ & $110.6(2)$ \\
$\mathrm{C}(2)-\mathrm{C}(1)-\mathrm{C}(5)$ & $60.5(2) \mathrm{O}(4)-\mathrm{C}(8)-\mathrm{C}(9)$ & $109.1(2)$ \\
$\mathrm{N}-\mathrm{C}(4)-\mathrm{C}(6)$ & $112.0(2) \mathrm{C}(11)-\mathrm{C}(8)-\mathrm{C}(9)$ & $113.5(3)$ \\
\hline
\end{tabular}

化合物 R1 和 $\mathbf{R} 1$ '单晶均属于单斜晶系 $P 2(1) / n$ 空间 
群. 由 $\mathbf{R} 1$ 的晶体结构图 6 可以看出, 以 $\mathrm{N}$ 原子所在的 五元吡咯环为平面, $\mathrm{N}$ 原子的左侧 $\mathrm{C}(1)$ 羧基与其右侧 $\mathrm{C}(6)$ 亚甲基处于异侧, $\mathbf{R} 1$ 属反式构型, 即为化合物 $(1 R, 3 S, 5 R)$-2-叔丁氧羰基-2-氮杂双环 [3.1.0]己烷-3-甲 酸; 相应的 R1'的晶体结构图 7 也能明显地观察出 $\mathrm{N}$ 原 子左侧 $\mathrm{C}(5)$ 亚甲基与其右侧 $\mathrm{C}(6)$ 羧基处于同侧, $\mathbf{R} 1$ '属 顺式构型，即为化合物 $(1 S, 3 S, 5 S)$-2-叔丁氧羰基-2-氮杂 双环[3.1.0]己烷-3-甲酸.

\section{2 结论}

以谷氨酸为起始原料, 经氨基保护、环合、还原、 西蒙斯一史密斯反应、水解等 5 步反应高立体选择性合 成目标化合物 $(1 R, 3 S, 5 R)$-2-叔丁氧羰基-2-氮杂双环 [3.1.0]己烷-3-甲酸(R1) 和 $(1 S, 3 S, 5 S)$-2-叔丁氧羰基-2-氮 杂双环[3.1.0]己烷-3-甲酸(R1'), 并探讨了 DMAP 催化 环合的最优条件，推测了西蒙斯一史密斯反应的反应历 程，最后得到了目标化合物 R1 和 R1'的单晶.

\section{3 实验部分}

\section{1 仪器与试剂}

北京泰克仪器有限公司生产的X-4 数字显示显微熔 点测定仪; Bruker 400 型核磁共振仪, 以 TMS 为内标氛 代氯仿 $\left(\mathrm{CDCl}_{3}\right)$ 或者気代二甲 $\left(\mathrm{DMSO}-d_{6}\right)$ 亚砜; 美国 Varian 公司生产的 Varian 1200 型高效液相色谱仪 (HPLC) Kromasil-C18 反相柱 $(4.6 \mathrm{~mm} \times 250 \mathrm{~mm}, 5 \mu \mathrm{m})$; 申光 SGW-1 自动旋光仪. 所有溶剂均为分析纯, 甲苯、 四氢呋喃经金属钠回流干燥, 使用前蒸出. 薄层色谱 (TLC)使用青岛海洋化工分厂生产的硅胶(200～300 目).

\section{2 实验方法}

\subsubsection{Boc- $D$-谷氨酸 $(\mathbf{R 5})$ 的制备}

$2000 \mathrm{~mL}$ 四口烧瓶中加入 $D$-谷氨酸 $(\mathbf{R 6})(100 \mathrm{~g}$, $0.70 \mathrm{~mol}) 、 1000 \mathrm{~mL} \mathrm{NaOH}$ 水溶液 $(85 \mathrm{~g}, 2.1 \mathrm{~mol})$, 冷却 到 $0{ }^{\circ} \mathrm{C}$, 加入二碳酸二叔丁酯 $(180 \mathrm{~g}, 1.0 \mathrm{~mol})$, 常温反 应 $20 \mathrm{~h}^{[25,26]}$. 用 $3 \mathrm{~mol} / \mathrm{L}$ 的 $\mathrm{HCl}$ 溶液调节 $\mathrm{pH}$ 至 3 . 乙酸 乙酯萃取三次, 无水硫酸钠干燥, 过滤, 旋蒸, 得到化 合物 R5 146 g, 收率 87\%. m.p. 109 111 ${ }^{\circ} \mathrm{C}$ (lit. ${ }^{[26]}$ $\left.113 \sim 114{ }^{\circ} \mathrm{C}\right)$. Boc- $L$-谷氨酸(S5)的合成方法同 $\mathbf{R 5}$.

\subsection{2 (R)-5-氧代吡咯-1,2-二甲酸二叔丁酯 $(\mathbf{R 4})$ 的制} 备

将 Boc- $D$-谷氨酸(R5) $(10 \mathrm{~g}, 41 \mathrm{mmol})$ 溶于 $100 \mathrm{~mL}$ 乙酸乙酯中, 再加入吡啶 $(3.2 \mathrm{~g}, 41 \mathrm{mmol})$ 、二碳酸二叔 丁酯 $(36 \mathrm{~g}, 163 \mathrm{mmol}), 35{ }^{\circ} \mathrm{C}$ 下搅拌反应 $15 \mathrm{~h}$. 冷却至 20 ${ }^{\circ} \mathrm{C}$, 加入 DMAP $(2.0 \mathrm{~g}, 16 \mathrm{mmol})$, 室温下摚拌反应 24

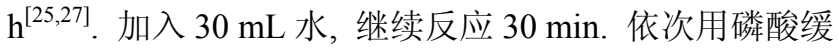

冲溶液、饱和碳酸氢钠水溶液洗涤 3 次, 无水硫酸钠干 燥, 过滤, 浓缩溶剂, 得到棕黄色黏稠的油状物, 用石 油醚和乙酸乙酯重结晶, 得到淡黄色固体 $\mathbf{R 4} 7.1 \mathrm{~g}$, 收 率 67\%. m.p. $54 \sim 56{ }^{\circ} \mathrm{C}$ (lit. ${ }^{[25]} 53 \sim 54{ }^{\circ} \mathrm{C}$ ); $[\alpha]_{\mathrm{D}}^{12}+$ 46.1 (c $\left.0.2, \mathrm{CH}_{3} \mathrm{CH}_{2} \mathrm{OH}\right) ;{ }^{1} \mathrm{H}$ NMR $\left(\mathrm{CDCl}_{3}, 400 \mathrm{MHz}\right) \delta$ : $1.48(\mathrm{~s}, 9 \mathrm{H}), 1.50(\mathrm{~s}, 9 \mathrm{H}), 1.96 \sim 2.01(\mathrm{~m}, 1 \mathrm{H}), 2.23 \sim 2.32$ (m, $1 \mathrm{H}), 2.42 \sim 2.48(\mathrm{~m}, 1 \mathrm{H}), 2.58 \sim 2.62(\mathrm{~m}, 1 \mathrm{H}), 4.63$ (dd, $J=9.5,2.5 \mathrm{~Hz}, 1 \mathrm{H}) ;{ }^{13} \mathrm{C}$ NMR $\left(\mathrm{CDCl}_{3}, 100 \mathrm{MHz}\right) \delta$ : $173.4,170.3,149.3,82.7,77.0,59.5,31.0,27.8,21.6$; ESIMS $m / z: 308.2\left(\mathrm{M}+\mathrm{Na}^{+}\right)$.

$(R)$-5-氧代吡咯-1,2-二甲酸二叔丁酯(S4)的合成与 R4 合成方法类似. 且 $[\alpha]_{\mathrm{D}}^{12}-46.7\left(c \quad 0.2, \mathrm{CH}_{3} \mathrm{CH}_{2} \mathrm{OH}\right)$ [lit. $\left.{ }^{[28]}-36.7\left(c 1, \mathrm{CHCl}_{3}\right)\right]$.

3.2.3 (R)-2,3-二氢- $1 H$ - 吡咯-1,2-二甲酸二叔丁酯 (R3)的制备

将 R4 (10 g, $35 \mathrm{mmol})$ 溶于 $200 \mathrm{~mL}$ 无水四氢呋喃中, 冷却至 $-75{ }^{\circ} \mathrm{C}$, 在氮气保护下缓慢加入 $35 \mathrm{~mL}$ 二异丁 基氢化铝(DIBAL, $1.5 \mathrm{~mol} / \mathrm{L}$ 的甲苯溶液)，摚拌反应 $2 \mathrm{~h}$. 再依次加入三氟乙酸 $(2.1 \mathrm{~g}, 18 \mathrm{mmol}) 、$ 三乙胺 $(21 \mathrm{~g}, 0.21$ $\mathrm{mol})$ 和三氟乙酸䣲 $(10 \mathrm{~g}, 48 \mathrm{mmol})$, 继续摚拌反应 $1.5 \mathrm{~h}$, 缓慢升温至室温 ${ }^{[29,30]}$. 将反应液加入到 $1 \mathrm{~mol} / \mathrm{L}$ 的柠檬 酸溶液中, 分液, 有机相分别用饱和碳酸氢钠水溶液和 饱和氯化钠水溶液洗涤两次, 无水硫酸钠干燥, 柱层析 提纯后得 $8.6 \mathrm{~g} \mathrm{R3}$, 收率 $90 \% .{ }^{1} \mathrm{H}$ NMR $\left(\mathrm{CDCl}_{3}, 400\right.$ MHz) $\delta: 1.47$ (s, 9H), 1.47 (s, 9H), $2.62(\mathrm{t}, J=8.2 \mathrm{~Hz}, 1 \mathrm{H})$, $2.99 \sim 3.09(\mathrm{~m}, 1 \mathrm{H}), 4.42 \sim 4.52(\mathrm{~m}, 1 \mathrm{H}), 4.87 \sim 4.92(\mathrm{~m}$, $1 \mathrm{H}), 6.49 \sim 6.64(\mathrm{~m}, 1 \mathrm{H}) ;{ }^{13} \mathrm{C} \mathrm{NMR}\left(\mathrm{CDCl}_{3}, 100 \mathrm{MHz}\right) \delta$ : 171.6, 153.3, 130.0, 104.8, 88.3, 82.5, 60.0, 27.9, 26.8. Anal. calcd for $\mathrm{C}_{14} \mathrm{H}_{23} \mathrm{NO}_{4}$ : C 62.43, H 8.61, N 5.20; found C 62.54, H 8.66, N 5.14.

$(S)$-2,3-二氢-1 $H$-吡咯-1,2-二甲酸二叔丁酯(S3) 的合 成与化合物 $\mathbf{R 3}$ 的合成方法类似.

$3.2 .4(1 R, 3 R, 5 R)-2$-氮杂双环 [3.1.0]己烷-2,3-二甲酸 二叔丁酯 $(\mathbf{R 2})$ 和 $(1 S, 3 R, 5 S)$-2-氮杂双环[3.1.0] 己烷2,3-二甲酸二叔丁酯 $\left(\mathbf{R 2}^{\prime}\right)$ 的制备

$500 \mathrm{~mL}$ 的四口烧瓶中加入化合物 $(R)-2,3-$ 二氢- $1 H$ 吡咯-1,2-二甲酸二叔丁酯 $(\mathbf{R} 3)(25 \mathrm{~g}, 93 \mathrm{mmol})$, 氮气保 护, 加入 $80 \mathrm{~mL}$ 三氟甲苯和氯碘甲烷 $(39 \mathrm{~g}, 0.22 \mathrm{~mol}$ ), 冷却至 $-20{ }^{\circ} \mathrm{C}$, 加入二乙基锌 $(186 \mathrm{~mL}, 1.1 \mathrm{~mol} / \mathrm{L}$ 甲苯 溶液), 反应 $20 \mathrm{~h}^{[31,32]}$. 将反应液滴入柠檬酸水溶液( 213 $\mathrm{mL}, 1 \mathrm{~mol} / \mathrm{L})$ 中, 分出有机相, 分别用饱和碳酸氢钠水 溶液和饱和氯化钠水溶液洗涤, 无水硫酸镁干燥, 过滤, 浓缩溶剂, 此步两种异构体可不分离. HPLC 测得两者 比例 $\left(\mathbf{R 2}^{\prime}: \mathbf{R 2}\right)$ 为 $6: 1$, 总收率 73\%.

R2: ${ }^{1} \mathrm{H}$ NMR $\left(\mathrm{CDCl}_{3}, 400 \mathrm{MHz}\right) \delta: 0.70 \sim 0.72(\mathrm{~m}$, 
$1 \mathrm{H}), 0.86 \sim 0.89(\mathrm{~m}, 1 \mathrm{H}), 0.89 \sim 1.25(\mathrm{~m}, 1 \mathrm{H}), 1.45(\mathrm{~s}$, $9 \mathrm{H}), 1.44(\mathrm{~s}, 9 \mathrm{H}), 1.97 \sim 2.01(\mathrm{~m}, 1 \mathrm{H}), 2.47 \sim 2.61(\mathrm{~m}$, $1 \mathrm{H}), 3.40 \sim 3.55(\mathrm{~m}, 1 \mathrm{H}, \mathrm{CH}), 4.36 \sim 4.49(\mathrm{~m}, 1 \mathrm{H})$. Anal. calcd for $\mathrm{C}_{15} \mathrm{H}_{25} \mathrm{NO}_{4}$ : C 63.58, $\mathrm{H} \mathrm{8.89,} \mathrm{N}$ 4.94; found $\mathrm{C}$ 63.50, H 8.87, N 4.92.

R2': ${ }^{1} \mathrm{H}$ NMR $\left(\mathrm{CDCl}_{3}, 400 \mathrm{MHz}\right) \delta: 0.78 \sim 0.79(\mathrm{~m}$, $1 \mathrm{H}), 0.80 \sim 0.89(\mathrm{~m}, 1 \mathrm{H}), 1.24 \sim 1.28(\mathrm{~m}, 1 \mathrm{H}), 1.48(\mathrm{~s}$, $9 \mathrm{H}), 1.46(\mathrm{~s}, 9 \mathrm{H}), 2.01 \sim 2.05(\mathrm{~m}, 1 \mathrm{H}), 2.49 \sim 2.53(\mathrm{~m}$, $1 \mathrm{H}), 3.46 \sim 3.58(\mathrm{~m}, 1 \mathrm{H}), 4.40 \sim 4.52(\mathrm{~m}, 1 \mathrm{H})$. Anal. calcd for $\mathrm{C}_{15} \mathrm{H}_{25} \mathrm{NO}_{4}$ : $\mathrm{C} 63.58, \mathrm{H} 8.89, \mathrm{~N} 4.94$; found $\mathrm{C} 63.38, \mathrm{H}$ 8.79, N 4.94 .

$(1 S, 3 S, 5 S)$-2-氮杂双环 [3.1.0]己烷-2,3-二甲酸二叔 丁酯 $(\mathbf{S 2})$ 和 $(1 R, 3 S, 5 R)-2$-氮杂双环 [3.1.0]己烷-2,3-二甲 酸二叔丁酯 $\left(\mathbf{S 2}^{\prime}\right)$ 的合成步骤与 $\mathbf{R 2}$ 和 $\mathbf{R 2}$ '合成方法类似. 3.2.5 (1R,3S,5R)-2-叔丁氧羰基-2-氮杂双环[3.1.0]己 烷-3-甲酸(R1)和(1S,3S,5S)-2-叔丁氧羰基-2-氮杂双 环[3.1.0]已烷-3-甲酸(R1')的制备

将 R2 和 R2'的混合物( $10 \mathrm{~g}, 35 \mathrm{mmol}$ )溶解于 100 $\mathrm{mL}$ 异丙醇溶液中, 加入 $300 \mathrm{~mL}$ 甲基叔丁基醚溶液, 加 热至 $35{ }^{\circ} \mathrm{C}, 2.5 \mathrm{~h}$ 内缓慢加入氢氧化钠 $(6.0 \mathrm{~g}, 88 \mathrm{mmol})$, 反应 $1 \mathrm{~h}$ 后, 加入 $2 \mathrm{~mL}$ 的水, 继续摚拌反应 $15 \mathrm{~h}^{[33]}$. 将 溶剂旋干, 加入 $100 \mathrm{~mL}$ 水和 $200 \mathrm{~mL}$ 甲基叔丁基醚, 用 $1 \mathrm{~mol} / \mathrm{L}$ 的盐酸水溶液调节 $\mathrm{pH}$ 至 3 , 甲基叔丁醚萃取, 无水硫酸镁干燥, 浓缩有机相, 得到混合物 $7.2 \mathrm{~g}$, 柱层 析 $[V$ (石油醚) $: V$ (乙酸乙酯 $)=10: 1$ ]分离提纯得白色固 体 R1 和 R1', 分别为 $4.8 \mathrm{~g}$ 和 $1.2 \mathrm{~g}$, 收率 $74.7 \%$.

R1: m.p. $134 \sim 136{ }^{\circ} \mathrm{C} ; \quad[\alpha]_{\mathrm{D}}^{12}-80.2$ (c 0.2 , $\left.\mathrm{CH}_{3} \mathrm{CH}_{2} \mathrm{OH}\right) ;{ }^{1} \mathrm{H}$ NMR $\left(\mathrm{CDCl}_{3}, 400 \mathrm{MHz}\right) \delta: 0.46 \sim 0.87$ $(\mathrm{m}, 2 \mathrm{H}), 1.36(\mathrm{~s}, 9 \mathrm{H}), 1.58 \sim 1.83(\mathrm{~m}, 1 \mathrm{H}), 2.10 \sim 2.29(\mathrm{~m}$, 2H), $3.18 \sim 3.33(\mathrm{~m}, 1 \mathrm{H}), 3.89$ (t, $J=12.68,1 \mathrm{H}), 12.45$ (s, $1 \mathrm{H}) ;{ }^{13} \mathrm{C} \mathrm{NMR}\left(\mathrm{CDCl}_{3}, 100 \mathrm{MHz}\right) \delta: 172.9,154.0,78.3$, 59.5, 39.4, 37.1, 28.9, 14.5, 13.9. Anal. calcd for $\mathrm{C}_{11} \mathrm{H}_{17} \mathrm{~N}-$ $\mathrm{O}_{4}$ : C 58.14, H 7.54, N 6.16; found C 58.04, H 7.53, N 6.10; ESIMS $m / z$ : $228.2\left(\mathrm{M}+\mathrm{H}^{+}\right)$.

R1': m.p. $134 \sim 136{ }^{\circ} \mathrm{C} ; \quad[\alpha]_{\mathrm{D}}^{12}-43.7$ (c 0.2, $\left.\mathrm{CH}_{3} \mathrm{CH}_{2} \mathrm{OH}\right) ;{ }^{1} \mathrm{H}$ NMR $\left(\mathrm{CDCl}_{3}, 400 \mathrm{MHz}\right) \delta: 0.62 \sim 0.74$ $(\mathrm{m}, 2 \mathrm{H}), 1.46(\mathrm{~s}, 9 \mathrm{H}), 1.49 \sim 1.54(\mathrm{~m}, 1 \mathrm{H}), 1.84 \sim 1.92(\mathrm{~m}$, $1 \mathrm{H}), 2.48 \sim 2.60(\mathrm{~m}, 1 \mathrm{H}), 3.31 \sim 3.34(\mathrm{~m}, 1 \mathrm{H}), 4.41 \sim 4.43$ $(\mathrm{m}, 1 \mathrm{H}), 12.56(\mathrm{~s}, 1 \mathrm{H}) ;{ }^{13} \mathrm{C} \mathrm{NMR}\left(\mathrm{CDCl}_{3}, 100 \mathrm{MHz}\right) \delta$ : 174.7, 153.4, 78.8, 59.0, 39.2, 36.7, 28.0, 14.8, 12.1. Anal. calcd for $\mathrm{C}_{11} \mathrm{H}_{17} \mathrm{NO}_{4}$ : C 58.14, H 7.54, N 6.16; found $\mathrm{C}$ 58.24, H 7.52, N 6.10; ESIMS $m / z$ : $228.2\left(\mathrm{M}+\mathrm{H}^{+}\right)$.

$(1 S, 3 R, 5 S)$-2-叔丁氧羰基-2-氮杂双环 [3.1.0]己烷-3甲酸 $(\mathbf{S 1})$ 和 $(1 R, 3 R, 5 R)-2$-叔丁氧羰基-2-氮杂双环 [3.1.0] 己烷-3-甲酸 $\left(\mathbf{S 1}^{\prime}\right)$ 的合成方法与 $\mathbf{R} 1$ 和 $\mathbf{R} 1^{\prime}$ '的合成方法类
似.

\subsubsection{R1 和 R1' '晶体结构的确定}

将 $\mathbf{R} 1$ 溶于乙酸乙酯溶液中, 滴加四氢呋喃, 采用 自然挥发法得到无色晶体, 选取 $0.30 \mathrm{~mm} \times 0.31 \mathrm{~mm} \times$ $0.25 \mathrm{~mm}$ 的晶体在用石墨单色器单色化的 $\mathrm{Mo} \mathrm{K} \alpha(\lambda=$ $0.71073 \AA$ )射线为辅射光源，在 $293 \mathrm{~K}$ 温度下，以 $\omega / 2 \theta$ 扫描方式在 $9^{\circ}<\theta<13^{\circ}$ 范围内, 共收集到 2605 个衍射 点, 其中 2263 为独立衍射强度数据, $R_{\mathrm{int}}=0.028$. 晶体结 构通过 SHELXS97 ${ }^{[34]}$ 程序直接法解出, 用 Fourier 合成 连续迭代得到非氢原子坐标, 对非氢原子坐标及各向异 性热参数以最小二乘法处理, 采用理论加氢, 最后非权 重和权重一致性因子 $(R$ 和 $w R)$ 分别为 0.0550 和 0.1518 . 晶体修正采用 SHELXL97 程序. 晶体数据已存于英国 剑桥数据中心(CCDC: 982318).

R1'采用类似方法得到单晶, 且用同样方法对单晶 进行了精修. 晶体数据已经存于英国剑桥数据中心 (CCDC: 982319).

\section{References}

[1] Abdel-Magid, A. M. Chem. Lett. 2012, 3, 699.

[2] Zhang, X.-P. Shanghai Med. Pharm. 2002, 23, 265 (in Chinese). (张小平, 上海医药, 2002, 23, 265.)

[3] Bender, J. A.; Hewawasam, P.; Kadow, J. F.; Lopez, O. D.; Meanwell, N. A.; Nguyen, V. N.; Romine, J. L.; Snyder, L. B.; St. Laurent, D. R.; Wang, G.; Xu, N.; Belema, M. WO 117635, 2010 [Chem. Abstr. 2010, 153, 1283826].

[4] Belema, M.; Good, A. C.; Goodrich, J.; Kakarla, R.; Li, G.; Lopez, O. D.; Nguyen, V. N.; Kapur, J.; Qiu, Y.; Romine, J. L.; St. Laurent, D. R.; Serrano-Wu, M.; Snyder, L. B.; Yang, F. WO 017401, 2010 [Chem. Abstr. 2010, 152, 175961].

[5] Belema, M.; Romine, J. L.; Nguyen, V. N.; Wang, G.; Lopez, O. D.; St. Laurent, D. R.; Chen, Q.; Bender, J. A.; Yang, Z.; Hewawasam, P.; Xu, N.; Meanwell, N. A.; Easter, J. A.; Su, B.-N.; Smith, M. J. WO 075439, 2011 [Chem. Abstr. 2011, 155, 785331].

[6] Vu, T. C.; Brzozowski, D. B.; Fox, R.; Godfrey, J. D., Jr.; Hanson, R. L.; Kolotuchin, S. V.; Mazzullo, J. A., Jr.; Patel, R. N.; Wang, J.; Wong, K.; Yu, J. Zhu, J.; Magnin, D. R.; Augeri, D. J.; Hamann, L. G. WO 052850, 2004 [Chem. Abstr. 2004, 141, 515478].

[7] Aissaoui, H.; Boss, C.; Gude, M.; Koberstein, R.; Sifferlen, T.; Trachsel, D. WO 081399, 2008 [Chem. Abstr. 2008, 149, 832909].

[8] Neitz, R. J.; Troung, A. P.; Galemmo, R. A.; Ye, X. M.; Sealy, J.; Adler, M.; Bowers, S.; Beroza, P.; Anderson, J. P.; Aubele, D. L.; Artis, D. R.; Hom, R. K.; Zhu, Y.-L. WO 048129, 2012 [Chem. Abstr. 2012, 156, 534299].

[9] Borriello, M.; Pucci, S.; Stasi, L. P.; Rovati, L. WO 004290, 2013 [Chem. Abstr. 2013, 158, 47589].

[10] Ren, J.; Feng, D.-J.; Li, Y.; Huang, J.-X. J. Hubei Correspondence Univ. 2000, 22, 371 (in Chinese). (任君, 冯代军, 李焰, 黄锦霞, 湖北大学学报, 2000, 22, 371.)

[11] Yu, H.; Ning, Z.-X. Fine Chem. 2006, 23, 145 (in Chinese). (于辉, 宁正祥, 精细化工, 2006, 23, 145.)

[12] She, Z.-G.; Chen, Y.-P.; Zhang, Q.-R. Wang, G.-J. Chem. Reag. 2001, 23, 110 (in Chinese).

(余志刚, 陈育平, 张歧荣, 王公纪, 精细化工, 2001, 23, 110.)

[13] Liang, Y.; Gao, S.-H.; Chen, S.-H. J. Tianjin Institute Technol. 
2001, 17(6), 18 (in Chinese).

(梁娅，高素华，陈苏战，天津理工学院学报, 2001, 17(6), 18.)

[14] Huang, N.-R.; Chen, Z.-R.; Hou, X.-L. Chin. J. Org. Chem. 1987, 7, 400 (in Chinese).

(黄乃正, 陈展荣, 侯雪龙, 有机化学, 1987, 7, 400.)

[15] Simmons, H. E.; Cairns, T. L.; Vladuchick, S. A.; Hoiness, C. M. Org. React. 1973, 20, 1.

[16] Girard, C.; Conia, J. M. J. Chem. Res. 1978, 182.

[17] Grieco, P. A.; Oguri, T.; Wang, C.-L. J.; Williams, E. J. Org. Chem. 1977, 42, 4113.

[18] Simmons, H. E.; Smith, R. D. J. Am. Chem. Soc. 1959, 81, 4256.

[19] Takahashi, H.; Yoshioka, M.; Ohno, M.; Kobayashi, S. Tetrahedron Lett. 1992, 33, 2575.

[20] Eric, L.; Sebastien, R. G.; Andre, B. C. Org. Lett. 2004, 16, 1490.

[21] Hall, C. R.; Inch, T. D. J. Chem. Soc. 1979, 4, 1104.

[22] Tang, C.-C.; Wu, G.-P. Sci. China 1986, 6, 584 (in Chinese). (唐除痴，吴桂萍，中国科学, 1986, 6, 584.)

[23] Tang, C.-C.; Zhang, W.-J.; Zhang, D.-C. Chem. J. Chin. Univ. 1992, 13, 1406 (in Chinese).

(唐除痴, 张纬吉, 张殿冲, 高等学校化学学报, 1992, 13, 1406.)

[24] Parr, I. B.; Boehlein, S. K.; Dribben, A. B.; Schuster, S. M.;
Richards, N. G. J. Med. Chem. 1996, 39, 2367.

[25] Gross, U.; Nieger, M.; Brase, S. Org. Lett. 2009, 11, 4740.

[26] Zhdanko, A. G.; Gulevich, A. V.; Nenajdenko, V. G. Tetrahedron 2009, 65, 4692.

[27] Hanessian, S.; Reinhold, U.; Saulnier, M.; Claridge, S. Med. Chem. 1998, 8, 2123.

[28] Barbara, G.; Anthony, S.; Craig, A. T. Biochemistry 2003, 42, 7836.

[29] Hanessian, S.; Reinhold, U.; Gentile, G. J. Angew. Chem., Int. Ed. 1997, 36, 1881.

[30] Howard, E. G., Jr.; Kotch, A.; Lindsey, R. V., Jr.; Putnam, R. E. J. Am. Chem. Soc. 1958, 80, 3924.

[31] Garcia, A. L. L.; Correia, C. R. D. Tetrahedron Lett. 2003, 44, 1553.

[32] Hiroaki, S.; Tsutomu, K. Angew. Chem., Int. Ed. 2008, 47, 2450.

[33] Galemmo, R. A., Jr.; Artis, D. R.; Ye, X. M.; Aubele, D. L.; Truong, A. P.; Bowers, S.; Hom, R. K.; Zhu, Y.-L.; Neitz, R. J.; Sealy, J.; Adler, M.; Beroza, P.; Anderson, J. P. WO 079118, 2011 [Chem. Abstr. 2013, 155, 47589].

[34] Shelsrick, G. M. SHELXS297, Program for the Refinement of Crystal Structures, University of Göttingen, Germany, 1997.

(Li, L.; Fan, Y.) 